\title{
Tumor Necrosis Factor Participates in the Pathogenesis of Acute Immune Complex Alveolitis in the Rat
}

\author{
Jeffrey S. Warren, Karen R. Yabroff, Daniel G. Remick, Steven L. Kunkel, Stephen W. Chensue, \\ Robin G. Kunkel, Kent J. Johnson, and Peter A. Ward \\ Department of Pathology, The University of Michigan Medical School, Ann Arbor, Michigan 48109-0602
}

\begin{abstract}
We have examined the role of intrapulmonary TNF in a rat model of acute immune complex-triggered alveolitis. Intratracheal instillation of IgG anti-bovine serum albumin (anti-BSA) followed by intravenous infusion of BSA results in acute alveolitis. Over the 4-h course of evolving lung injury, a 10-fold increase in TNF activity occurred in bronchoalveolar lavage (BAL) fluid. Immunohistochemical analysis of lung sections and BAL cells revealed that alveolar macrophages are the chief source of TNF. Antibodies that specifically neutralize rat TNF activity were raised in rabbits immunized with recombinant mouse TNF $\alpha$. When administered into the lungs with anti-BSA, anti-TNF resulted in a marked reduction (up to $61 \%$ ) in lung injury. Intratracheal instillation of exogenous TNF alone, or in combination with anti-BSA, resulted in an increase in lung injury compared to controls. Morphometric analysis and measurements of myeloperoxidase activities in whole lung extracts from rats treated with anti-TNF revealed a marked reduction in neutrophils compared to positive controls. The anti-TNF antibody preparation did not inhibit in vitro complement activation or diminish neutrophil chemotactic activity present in activated rat serum. These data indicate that intrapulmonary TNF activity is required for the full development of acute immune complex-triggered alveolitis, that alveolar macrophages are the primary source of this cytokine, and that TNF participates in the pathogenesis of immune complex alveolitis through a mechanism involving neutrophil recruitment.
\end{abstract}

\section{Introduction}

Tumor necrosis factor (TNF) ${ }^{1}$ is a hormone-like polypeptide that possesses a wide range of metabolic, immunologic and inflammatory activities (reviewed in 1). Once attributed to the direct effects of bacterial endotoxin, many of the metabolic and physiologic derangements of endotoxic and septic shock

Address reprint requests to Dr. Warren, Department of Pathology, Box 0602, The University of Michigan Medical School, 1301 Catherine Road, Ann Arbor, MI 48109-0602.

Received for publication 6 February 1989 and in revised form 9 June 1989.

1. Abbreviations used in this paper: BAL, bronchoalveolar lavage; HPF, high power field; LPS, lipopolysaccharide; NCF, neutrophil chemotactic factor; TNF, tumor necrosis factor.

J. Clin. Invest.

(C) The American Society for Clinical Investigation, Inc.

$0021-9738 / 89 / 12 / 1873 / 10 \quad \$ 2.00$

Volume 84, December 1989, 1873-1882 are thought to be mediated by endogenous cytokines, notably TNF. Physiologically significant, even lethal, quantities of TNF have been detected in the sera of animals after infusion of endotoxin or viable bacteria $(2,3)$. Studies in several mammalian species have shown that most of the pathophysiologic manifestations of endotoxemic shock can be reproduced by injection of recombinant TNF $(2,4-7)$. Passive immunization of mice and rabbits with anti-TNF antibodies before infusion of lethal doses of endotoxin attenuates major pathophysiologic manifestations of endotoxic shock and reduces the incidence of endotoxin-mediated death $(4,8)$.

It has been proposed that locally regulated generation of TNF at sites of injury represents an important autocrine-paracrine control mechanism operative in acute inflammatory responses. In vitro studies suggest that TNF is chemotactic for neutrophils (9), induces their degranulation (10), induces their adhesion to endothelium (11), and stimulates their release of oxygen-derived metabolites $(10,12)$. TNF also activates macrophages resulting in IL-1 and $\mathrm{PGE}_{2}$ production (13), augmented cytotoxic potential $(14,15)$, and enhanced superoxide anion production after exposure to immune complexes (16). TNF has been shown to directly increase endothelial cell procoagulant activity by several mechanisms (17-21). TNF induces the synthesis of endothelial cell surface factors that increase neutrophil adherence to the endothelial surface through mechanism(s) dependent on subunits of the neutrophil membrane adhesion complex CDw18 (Mac-1/LFA-1/p150,95) (11, 22). Horvath et al. (23) have shown that intravenous infusion of human TNF into sheep results in increases in pulmonary vascular resistance, transvascular fluid filtration, and transvascular protein clearance. Both in vivo and in vitro studies suggest that TNF increases endothelial permeability to proteins through a neutrophil-independent mechanism (23). TNF has also been reported to trigger platelet-activating factor synthesis by endothelial cells (24). Recently, Strieter et al. (25) demonstrated that TNF can stimulate endothelial cell production of a 7,500-D neutrophil chemotactic factor (NCF) and mRNA transcripts that encode NCF.

Although TNF appears to be an important mediator in the shock syndrome caused by infusion of live bacteria or bacterial endotoxin and apparently modulates a variety of inflammatory cell functions, the role of TNF in compartmentalized acute inflammatory processes in vivo has been studied only preliminarily. A synergistic effect between TNF and IL-1 has been described in the induction of Shwartzman-like reactions (26) and in the induction of neutrophil emigration (27), while studies of neutrophil emigration triggered by intradermal endotoxin injections suggest that TNF $\alpha$ by itself does not mediate neutrophil emigration (28). In mice, intradermal injection of TNF $\alpha$ has been shown to trigger acute dermal inflammation (29). These studies serve to emphasize the prevailing uncertainties surrounding the role of TNF in acute inflammation. 
In the present study we examined the role of intrapulmonary TNF in the pathogenesis of acute immune complex-induced alveolitis in the rat. We sought to determine whether TNF is produced in the lung during the evolution of immune complex-induced lung injury, which pulmonary cells produce TNF, and to what extent this cytokine may contribute to the pathogenesis of acute alveolitis. The results of this study demonstrate that TNF is an important mediator of acute immune complex alveolitis and that it influences this process through a mechanism involving neutrophil recruitment.

\section{Methods}

Animal model of immune complex alveolitis. Male Long-Evans specific pathogen free rats (300-400 g; Charles River Breeding Laboratories, Inc., Wilmington, MA) were used for all studies. Intraperitoneal injections of ketamine $(2.5-5.0 \mathrm{mg} / 100 \mathrm{~g}$ body $\mathrm{wt}$ ) and sodium pentobarbital $(5 \mathrm{mg} / 100 \mathrm{~g}$ body wt) were given for sedation and anesthesia. Immune complex lung injury was induced as previously described $(30$, 31). Antibody solutions ( $250 \mu \mathrm{g}$ anti-BSA unless otherwise specified), recombinant mouse TNF $\alpha$ (Genzyme, Boston, MA), or mixtures of antibody and TNF, were instilled into the lungs via a tracheal cannula. In all cases, a final volume of $200 \mu \mathrm{l}$ was instilled into the lungs. Antigen (BSA, $10 \mathrm{mg}$ ) was injected intravenously. Rats were sacrificed at the indicated times and BAL fluid harvested (see below) or lung injury quantitated. Pulmonary injury was quantitated by permeability measurements as well as by morphometric analysis. Permeability indices were calculated by comparing the leakage of ${ }^{125} \mathrm{I}$-labeled bovine gamma globulin from the circulation into the lung to the counts per minute ${ }^{125} \mathrm{I}$-labeled colloid in $1 \mathrm{ml}$ of blood as previously described (30).

Collection of BAL fluid and isolation of alveolar macrophages. Alveolar macrophages were recovered from normal rats using a previously described modification of the method of Van Oud Alblas and Van Furth (32).

BAL contents for cytokine measurements and cellular function assays were collected using $5 \mathrm{ml}$ of $37^{\circ} \mathrm{C}$, serum-free RPMI 1640 . At least $90 \%$ of the administered fluid was always recovered, centrifuged $(400 \mathrm{~g} ; 7 \mathrm{~min})$ to remove cells, and stored at $-20^{\circ} \mathrm{C}$ before analysis.

Morphometric analysis of lung injury. Transmission electron micrographs were prepared from whole lungs fixed in $4 \%$ glutaraldehyde under constant pressure inflation $\left(25 \mathrm{~cm} \mathrm{H}_{2} \mathrm{O}\right.$ ), washed in $0.1 \mathrm{M}$ cacodylate buffer ( $\mathrm{pH} 7.3$ ), and embedded in plastic. 1- $\mu \mathrm{m}$-thick sections were stained with toluidine blue and processed for transmission electron microscopy by using a 401 transmission electron microscope (401; Philips Electronic Instruments, Mahwah, NJ). As previously described (33), morphometric analysis was carried out by an experienced electron microscopist (R. G. Kunkel) who was blinded to sample origin. For each condition five lung samples were examined. In each sample, 45-60 randomly selected $40 \times$ microscopic fields were analyzed.

Preparation of IgG anti-BSA. Rabbit (or mouse) IgG rich in antibody directed against BSA was used to induce lung injury and to form precipitating polyclonal IgG-BSA immune complexes. Antibody content and production of immune complexes have been previously described in detail (30). Briefly, IgG was prepared from hyperimmune serum by precipitation with saturated ammonium sulfate $(50 \%)$, followed by dialysis against 0.005 M PBS and passage through a DEAE cellulose column. Where indicated, antibody was heat denatured $\left(100^{\circ} \mathrm{C}, 20 \mathrm{~min}\right)$. The IgG anti-BSA and BSA (low endotoxin; ICN Biomedicals, Costa Mesa, CA) preparations contained $<0.02 \mathrm{ng} / \mathrm{ml}$ and $0.012 \mathrm{ng} / \mathrm{ml}$ of endotoxin activity, respectively, as estimated using the Limulus amebocyte lysate (LAL) assay (E-toxate; Sigma Chemical Co., St. Louis, MO). Antigen-antibody equivalence has previously been shown to occur at a 1:5 (wt/wt) ratio (30).
Preparation of $T N F$ and $I L-1$-rich conditioned medium from alveolar macrophages. Rat alveolar macrophages were suspended in RPMI 1640 (KC Biological, Lenexa, KS), supplemented with 100 $\mathrm{U} / \mathrm{ml}$ penicillin, $100 \mu \mathrm{g} / \mathrm{ml}$ streptomycin, $300 \mu \mathrm{g} / \mathrm{ml}$ fresh glutamine, and $10 \%$ heat-inactivated fetal calf serum (FCS; Gibo Laboratories, Grand Island, NY). Alveolar macrophages $\left(2 \times 10^{6} / \mathrm{ml}\right)$ were plated in 35-mm polystyrene dishes (Corning Glass Works, Corning, NY) and maintained in a humidified $5 \% \mathrm{CO}_{2}$ atmosphere at $37^{\circ} \mathrm{C}$ to allow macrophages to adhere. After $2 \mathrm{~h}$, the dishes were gently washed twice with serum-free $37^{\circ} \mathrm{C}$ RPMI 1640 and Escherichia coli lipopolysaccharide 0111:B4 (Sigma Chemical Co.) $(10 \mu \mathrm{g} / \mathrm{ml})$ was added to culture dishes. The dishes were maintained for $4 \mathrm{~h}$ in serum-free RPMI under the same conditions as above. After the incubation period, supernatants were collected, and aliquots assayed for IL-1 and TNF activity.

TNF assay. TNF activity was measured by means of modification of the procedure of Ruff and Gifford (34). Briefly, equal volumes of $\log _{2}$ dilutions (final volume $0.1 \mathrm{ml}$ ) of sample-containing media were made in 96-well microtiter plates (Costar, Cambridge, MA) containing target LM cells (mouse fibroblast cell line derived from NCTC clone 929; American Type Culture Collection) $\left(5 \times 10^{4}\right.$ cells per $\left.0.1 \mathrm{ml}\right)$ in the presence of actinomycin D (final concentration, $1 \mu \mathrm{g} / \mathrm{ml}$ ). In a separate set of wells, a standard consisting of serially diluted recombinant human TNF (Cetus Immune Laboratories, Palo Alto, CA) was added. The cells were incubated at $37^{\circ} \mathrm{C}$ for $18 \mathrm{~h}$, the supernatants discarded, and the remaining viable adherent cells washed with PBS and then stained by adding crystal violet $(0.2 \%$ in $2 \%$ ethanol) for 5 min. Microtiter plates were rinsed three times with PBS and allowed to air dry. The absorbance of each well was read at $620 \mathrm{~nm}$ with a MicroELISA Autoreader (BioTek Instruments, Winooski, VT). Units of TNF are defined as the reciprocal of the dilution at which $50 \%$ cytolysis occurs.

IL-1 assay. IL-1 levels were measured by means of a thymocyte coproliferation assay, as modified from the procedure of Mizel et al. (35). Briefly, $5 \times 10^{5}$ murine $\left(\mathrm{C}_{3} \mathrm{H} / \mathrm{HeJ}\right)$ thymocytes in $0.1 \mathrm{ml}$ of complete RPMI 1640 containing $2.5 \mu \mathrm{g} / \mathrm{ml}$ of phytohemagglutinin (Burroughs Welcome, Research Triangle Park, NC) were added to each well of a 96-well culture plate. Equal volumes of $\log _{2}$ dilutions of sample were added to the wells in triplicate. After $66 \mathrm{~h}$ incubation at $37^{\circ} \mathrm{C}$ in $5 \% \mathrm{CO}_{2} / 95 \%$ air, the thymocytes were pulsed with $0.5 \mu \mathrm{Ci}$ $\left[{ }^{3} \mathrm{H}\right]$ thymidine $(6.9 \mathrm{Ci} / \mathrm{nmol}$; ICN Nutritional Biochemicals, Irvine, CA) and were collected $6 \mathrm{~h}$ later on glass fiber strips by using an automatic cell harvester. $\left[{ }^{3} \mathrm{H}\right]$ Thymidine incorporation was determined by using a liquid scintillation counter. For standardization, data are expressed as counts per minute of $\left[{ }^{3} \mathrm{H}\right]$ thymidine incorporated by thymocytes that were incubated with a $1 / 32$ dilution of macrophage supernatant. In addition, a known recombinant human IL-1 $\beta$ standard (The Upjohn Co., Kalamazoo, MI) was assayed concomitantly with unknown samples. In the IL-1 assay, the unstimulated background response averaged $200 \mathrm{cpm}$, whereas the response to 0.25 unit of the commercially available standard averaged $2,700 \mathrm{cpm}$.

Preparation of IgG anti-TNF antibodies. Anti-TNF alpha was produced by immunization of rabbits with recombinant murine TNF as previously described (36). The resulting antiserum was affinity purified using a protein A Sepharose column (Sigma). Anti-TNF serum was diluted 1:1 with PBS (100 $\mathrm{mM}$ phosphate, $\mathrm{pH} 8.0,150 \mathrm{mM} \mathrm{NaCl})$ and applied slowly to the PBS-washed column. After extensive washing with $\mathrm{PBS}$, the column was stripped with $100 \mathrm{mM}$ sodium acetate buffer, $\mathrm{pH}$ 3.0. $1-\mathrm{ml}$ fractions were collected in tubes containing $50 \mu \mathrm{l}$ of $1 \mathrm{M}$ Tris buffer, $\mathrm{pH}$ 8.0. The affinity purified $\mathrm{IgG}$ fraction was then dialyzed against PBS and characterized as described below.

Western immunoblot analysis of anti-TNF. Recombinant murine IL-1 $\beta$ (100 ng), IL-1 $\alpha$ (200 ng), and TNF $\alpha$ (200 ng) were electrophoresed in $12.5 \%$ SDS-PAGE with a $7 \%$ stacking gel according to the method of Laemmli (37). The electrophoretically separated proteins were transblotted to nitrocellulose $(0.45 \mu \mathrm{m}$; Bio-Rad Laboratories, Richmond, CA) for 20 min at $24 \mathrm{~V}$ with a Genie transblot apparatus (Idea Scientific, Corvallis, OR). After transfer, the gel was blocked by 
treatment with Tween-Tris-buffered saline ([T-TBS], $20 \mathrm{mM}$ Tris$\mathrm{HCl}, \mathrm{pH} 7.5,500 \mathrm{mM} \mathrm{NaCl}, 0.05 \% \mathrm{vol} / \mathrm{vol}$ Tween) containing $5 \%$ lowfat milk and $1 \%$ normal goat serum for $1 \mathrm{~h}$. The gel was twice washed with T-TBS, followed by addition for $1 \mathrm{~h}$ of purified rabbit anti-mouse TNF at a final concentration of $1 \mu \mathrm{g} / \mathrm{ml}$. The gel was then washed twice with T-TBS followed by addition of biotinylated goat anti-rabbit IgG $(1: 3,000)$ for $1 \mathrm{~h}$. After two additional washes, streptavidin-alkaline phosphatase complex was added for $1 \mathrm{~h}$, the nitrocellulose washed, and bands developed. Biotinylated protein standards were used to assess molecular weights.

Immunohistochemical analysis of lung sections and BAL cells. Immunochemical analyses of lung sections and BAL cells were carried out using adaptations of previously described methods (36). In order to avoid potential binding of biotinylated secondary antibody (goat antirabbit) to the rabbit anti-BSA routinely used to induce alveolitis, mouse IgG anti-BSA was substituted. Comparison studies have shown rabbit and mouse IgG anti-BSA preparations to be indistinguishable in this model system (data not shown).

At the indicated times after induction of lung injury, a 3:1 mixture (vol/vol) of OCT embedding medium (Miles Laboratories, Naperville, IL) and PBS ( $10 \mathrm{mM}, \mathrm{pH} 7.4$ ) was instilled into whole rat lungs, which were then frozen $\left(-20^{\circ} \mathrm{C}\right)$. Frozen sections were fixed for $10 \mathrm{~min}$ in acetone, rehydrated with PBS, and fixed ( $3 \mathrm{~min}$ ) in absolute methanol. The slides were rinsed again with PBS and treated with a 1:50 dilution of normal goat serum for $10 \mathrm{~min}$ at $37^{\circ} \mathrm{C}$, then decanted and exposed to a 1:1,000 dilution of anti-TNF $\alpha$ or a similar dilution of nonimmune rabbit serum. After $20 \mathrm{~min}$ of incubation at $37^{\circ} \mathrm{C}$, the slides were rinsed three times with PBS, then overlaid with biotinylated goat antirabbit IgG (1:100) (Vector Laboratories, Burlingame, CA) and incubated another $10 \mathrm{~min}$, followed by three additional rinses with PBS. The slides were next treated with alkaline phosphatase labeled streptavidin and levamisole (endogenous alkaline phosphatase inhibitor), incubated again, rinsed three times, then overlaid with substrate chromogen (Kit I, Vector Red) for $5 \mathrm{~min}$ at $37^{\circ} \mathrm{C}$ to allow for color development. Mayer's hematoxylin was used as a counterstain.

In separate experiments, BAL cells were harvested $0,1,2.5$, and $4 \mathrm{~h}$ after initiation of lung injury. The cells were washed and suspended in serum-free RPMI 1640 at $10^{6}$ cells $/ \mathrm{ml}$. Cells $(250 \mu \mathrm{l})$ were then added to 8-well slide tissue culture chambers (Lab-Tek, Miles Laboratories) at $37^{\circ} \mathrm{C}$ in $5 \% \mathrm{CO}_{2}$, humidified atmosphere. After $1 \mathrm{~h}$, nonadherent cells were rinsed away with warm RPMI 1640. BAL cell monolayers were fixed and stained as described above except that the slides were treated with 3\% hydrogen peroxide solution to inactivate any remaining endogenous peroxidase activity. After treatment of the slides with biotinylated anti-rabbit IgG, the slides were treated with peroxidase-labeled streptavidin (Sigma Chemical Co.) and developed with 3-amino-9ethylcarbazole.

Whole lung myeloperoxidase activities. Whole lungs were homogenized with a Polytron homogenizer $(4 \times 10 \mathrm{~s}$ at a setting of 4$)$ using 6 $\mathrm{ml}$ of homogenization buffer. The homogenization buffer $(50 \mathrm{mM}$ phosphate, $\mathrm{pH} 6.0$ ) contained $0.5 \%$ hexadecyltrimethyl ammonium bromide (Sigma) and $5 \mathrm{mM}$ EDTA. Homogenized samples were then sonicated $(3 \times 10 \mathrm{~s}$ at a setting of 5$)$ and centrifuged $(3,000 \times g ; 30 \mathrm{~min})$ at $4^{\circ} \mathrm{C}$. Myeloperoxidase activity in supernatants was assayed by measuring the change in $\mathrm{A}_{460}$ resulting from decomposition of $\mathrm{H}_{2} \mathrm{O}_{2}$ in the presence of $o$-dianisidine (38).

Neutrophil chemotaxis and serum activation. Heparinized caval blood was collected from Long-Evans rats and the neutrophils isolated by centrifugation over a Histopaque-1077 gradient (Sigma) after sedimentation of red blood cells with hydroxy ethyl starch (6\% Volex; McGaw Laboratories, Glendale, CA) (39). Residual erythrocytes were removed by hypotonic lysis. The purified neutrophils $(83-86 \%$ by Wright stain morphology) were suspended at $1.5 \times 10^{6} / \mathrm{ml}$ in HBSS with calcium $(1.8 \mathrm{mM})$, magnesium $(0.8 \mathrm{mM})$ and $1 \%$ BSA. Chemotaxis was performed in blind multi-well Boyden chemotactic chambers (40). $225 \mu$ l HBSS was placed in the bottom well with either HBSS alone, zymosan $(0.1 \mathrm{mg} / \mathrm{ml})$ activated rat serum $(25 \mu \mathrm{l})$ or unactivated rat serum $(25 \mu \mathrm{l}$ ). A $3 \mu \mathrm{m}$ polycarbonate filter (polyvinylpyrrolidone

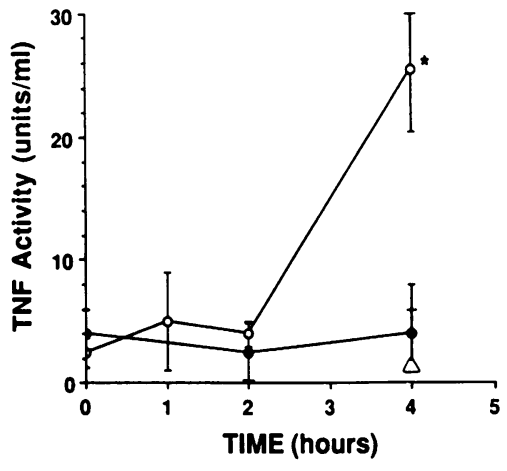

Figure 1. Elaboration of intrapulmonary (BAL fluid) TNF during the evolution of IgG immune complex-mediated lung injury. Rats were sacrificed at the indicated time points and lungs lavaged with serum-free RPMI 1640 ( $5 \mathrm{ml})$. BAL TNF activities were assayed from IgG immune complexinjured rats $(0)$, negative control rats (nonspecific intratracheal $\mathrm{IgG})(\bullet)$, and noninjured (normal) rats $(\Delta)$. The data expressed represent means \pm SEM of three experiments in which three to five animals were employed for each variable. For normal rats, three animals were employed in a single experiment. Data were analyzed by one-way analysis of variance with significance assigned for $P<0.05(46)$. * Indicates significant differences versus injured rats at time zero, negative control rats, and normal rats.

free; Nucleopore Corp., Pleasanton, CA) was placed in the assembly and $333 \mu \mathrm{l}$ of neutrophil suspension placed in the top chamber. The neutrophils were incubated at $37^{\circ} \mathrm{C}$ in a humidifed chamber. The filters were removed and stained with Diff-Quik (Baxter Corp., Chicago, IL). Locomotion indices were calculated as described by Maderazo et al. (41). Experiments addressing the effects of anti-TNF on serum activation were conducted similarly, except that varying concentrations of anti-TNF or control antibody were added to rat serum during exposure to zymosan. The resulting supernatants were then assayed for chemotactic activity.

\section{Results}

Elaboration of intrapulmonary TNF during IgG immune complex-induced lung injury. Bronchoalveolar lavage (BAL) fluids were examined for the presence of TNF as a function of time after airway instillation of anti-BSA and intravenous infusion of antigen. It is apparent that very little increase in TNF activity could be detected in the first $2 \mathrm{~h}$ (Fig. 1). After this time there was a rapid rise in BAL TNF activity; by $4 \mathrm{~h}$, TNF levels had increased 10-fold. In animals that received anti-BSA in the absence of antigen or heat-denatured anti-BSA in the presence

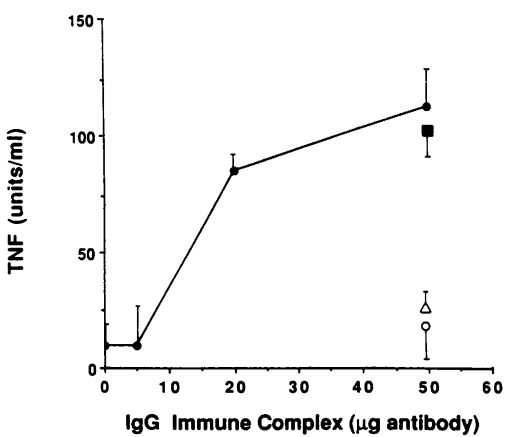

Figure 2. Preformed IgG immune complexes trigger release of TNF from isolated alveolar macrophages. Rat alveolar macrophages suspended in RPMI 1640 with $10 \%$ heat-inactivated fetal bovine serum were allowed to adhere to polystyrene culture dishes $(2$ $\times 10^{6} / 35 \mathrm{~mm}$ dish), washed, and incubated for $4 \mathrm{~h}$ in serum-free RPMI 1640 containing varying concentrations of preformed IgG immune complexes $(\bullet)$ in presence ( $(\boldsymbol{)})$ or absence of polymyxin B $(50 \mu \mathrm{g} / \mathrm{ml})$. Negative controls included equivalent concentrations of $\mathrm{IgG}$ alone $(0)$ or BSA alone $(\Delta)$. These data are expressed as means \pm SEM of three experiments in which duplicates or triplicates were employed for each variable. All values are normalized for adherent cell number. 

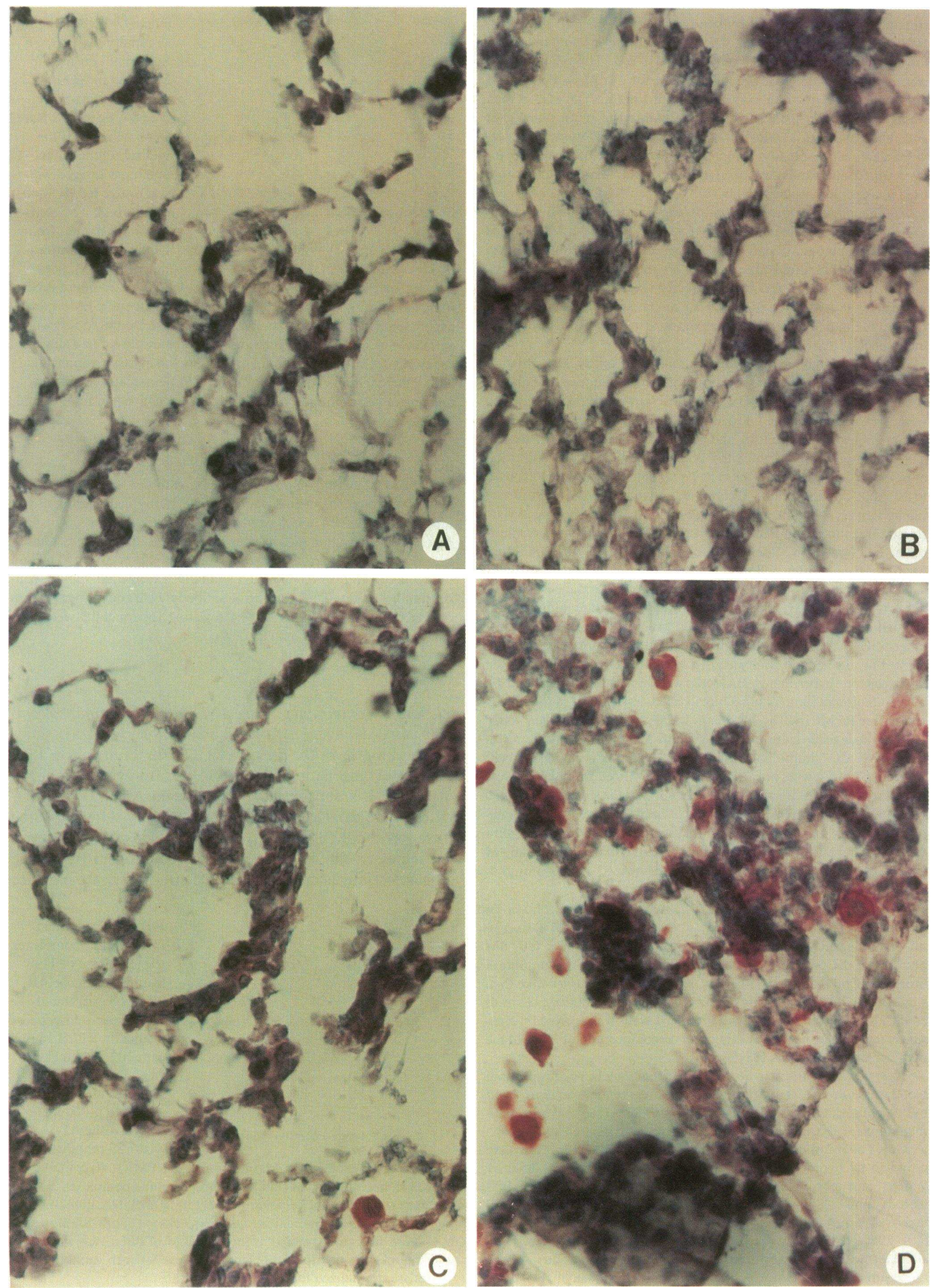
Table I. Immune Complex Alveolitis: Immunohistochemical Staining of BAL Cells for TNF*

\begin{tabular}{cc}
\hline Time & Percent positively staining cells ${ }^{\ddagger}$ \\
\hline$h$ & \\
0 & $5.6 \pm 5.5$ \\
1 & $3.0 \pm 1.0$ \\
2.5 & $13.0 \pm 6.0$ \\
4 & $25.2 \pm 13.0$
\end{tabular}

\footnotetext{
* BAL cells were obtained at the indicated times following induction of immune complex alveolitis. Cells were incubated in eight-well slide tissue culture chambers for $1 \mathrm{~h}$ before nonadherent cells were washed away.

‡ Controls consisted of identically prepared samples incubated with normal rabbit serum instead of anti-TNF. Less than $1 \%$ of control cells stained positively at each time point. At least 300 cells were counted for each condition.
}

of antigen (BSA), the BAL control at $4 \mathrm{~h}$ contained $<3 \mathrm{U} / \mathrm{ml}$ TNF (data not shown). These controls suggest that endotoxin contamination of the anti-BSA or BSA is not responsible for eliciting BAL fluid TNF and that the intrapulmonary elaboration of this cytokine is dependent on the functional integrity of the anti-BSA.

In vitro elaboration of TNF by isolated rat alveolar macrophages. Since alveolar macrophages represent the major resident mononuclear phagocyte population in the lung and are a likely source of intraalveolar TNF, we measured TNF secretion by isolated alveolar macrophages stimulated by immune complexes derived from the same reagents employed in vivo. As shown in Fig. 2, incubation of alveolar macrophages with increasing concentrations of preformed IgG immune complexes resulted in dose-dependent release of TNF. Incubation of macrophages with IgG alone or BSA alone resulted in minimal TNF release, suggesting that cell stimulation is due to the presence of immune complexes and not due to a factor such as bacterial lipopolysaccharide present in antigen or antibody preparations. Furthermore, addition of polymyxin B had no suppressive effect on immune complex-triggered TNF release, suggesting that endotoxin contamination is not responsible for macrophage activation.

Immunohistochemical localization of TNF production in acute alveolitis. Immunohischemical studies were carried out to determine the in vivo source(s) of intrapulmonary TNF elaboration during the development of immune complex alveolitis. Examination of lung sections obtained 0, 1, 2.5, and 4 $h$ after induction of lung injury revealed a progressive increase in the proportion of alveolar macrophages that stained positively for TNF (Fig. 3). At each time point, TNF activity appeared to be largely restricted to alveolar macrophages.

In a separate experiment, immune complex lung injury was induced and BAL cells were harvested after $0,1,2.5$, or 4 h. Samples of BAL cells were placed into 8-well slide tissue culture chambers, where after $1 \mathrm{~h}$, nonadherent cells were

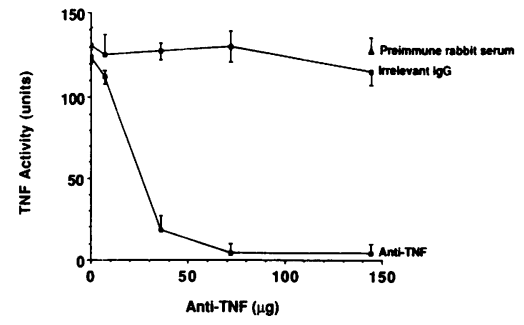

Figure 4. In vitro neutralization of TNF activity with affinity purified IgG anti-TNF $\alpha$. Rat TNF activity (where 1 unit $=50 \%$ lysis of murine LM cells [34]) was assayed in serum-free conditioned medium (RPMI 1640)

obtained from LPS-stimulated rat alveolar macrophages. Microtiter wells containing serial dilutions of conditioned medium and LM cells were incubated in the presence of preimmune rabbit serum $(\boldsymbol{\Delta})$ $(50 \mu)$, irrelevant affinity purified rabbit $\operatorname{IgG}(\varpi)$, or affinity purified anti-TNF $\alpha(\square)$. The data expressed represent the means \pm SEM of two experiments in which each variable was assayed in quadruplicate. In both experiments, aliquots of conditioned medium were obtained from the same stock. All LM cell cultures contained a final volume of $200 \mu \mathrm{l}$.

washed away. Immunohistochemical staining revealed a progressive increase in the percentage of alveolar macrophages that stained positively for TNF (Table I). These studies provide strong evidence that alveolar macrophages are the chief source of intrapulmonary TNF activity in evolving immune complex alveolitis.

In vitro neutralization of rat TNF activity with specific anti-TNF antibodies. Specific anti-murine TNF antibodies were raised in rabbits immunized with recombinant mouse TNF $\alpha$. Immunoblot analysis of the anti-TNF revealed reactivity with TNF and the absence of reactivity with either IL- $1 \alpha$ or IL-1 $\beta$ (data not shown). In order to permit analysis of the role of TNF in immune complex alveolitis we examined the capacity of this antibody preparation to specifically neutralize rat TNF activity.

Aliquots of serum-free TNF and IL-1-rich conditioned medium from rat alveolar macrophage cultures were used to test the neutralizing capacity of the anti-TNF antibody and to verify the absence of cross-reactivity with a potentially relevant cytokine, IL-1. Affinity purified IgG anti-TNF antibodies blocked rat TNF activity in a dose-dependent manner (Fig. 4). Equivalent concentrations of irrelevant affinity purified rabbit IgG had no neutralizing effect on rat TNF activity. Likewise, addition of heat-inactivated preimmune rabbit serum had no inhibitory effect on TNF activity. The negligible differences in TNF activity present in aliquots incubated with preimmune serum versus TNF activity present in aliquots not exposed to any added serum or antibody suggest that the presence of IgG per se does not influence measurements of TNF activity. Neither anti-TNF $(72 \mu \mathrm{g})$, irrelevant rabbit IgG $(72 \mu \mathrm{g})$, nor preimmune rabbit serum had any blocking effect on IL-1 activity present in conditioned medium.

Blockade of IgG immune complex alveolitis with anti-TNF antibodies. Intratracheal instillation of anti-TNF resulted in a $61 \%$ reduction in IgG immune complex induced lung injury (Fig. 5). Lung injury was quantitated by measuring increases in

Figure 3. Immunohistochemical localization of intrapulmonary TNF production in acute immune complex alveolitis. $A$ and $B$ reveal lung sections obtained 0 and $4 \mathrm{~h}$ after initiation of lung injury, respectively. These sections were incubated with normal rabbit serum (negative controls). $C$ and $D$ show lung sections obtained 0 and $4 \mathrm{~h}$ after initiation of lung injury, respectively. These sections were stained with anti-TNF antibody. $A, B, C$, and $D$ are $\times 162$, counterstained with Mayer's hematoxylin. 


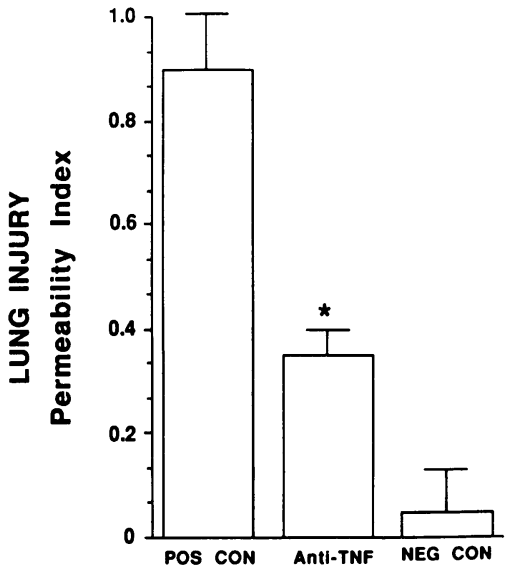

ure, the positive controls received nonspecific IgG. Negative control rats received intratracheal anti-BSA but no intravenous BSA. Lung injury was quantitated by determining permeability indices $4 \mathrm{~h}$ after instillation of anti-BSA as previously described (30). These data represent means \pm SEM of four experiments in which three to four rats per variable were employed. The data were analyzed by one-way analysis of variance with significance assigned for $P$ $<0.05 . *$ Indicates a significant difference vs. positive controls.

pulmonary vascular permeability as determined by leakage of ${ }^{125}$ I-labeled colloid into the airspace and lung parenchyma (30). Substitution of equivalent concentrations of heat-inactivated anti-TNF or nonspecific rabbit IgG for anti-TNF failed to suppress immune complex lung injury ( $<10 \%$ reduction) suggesting that the anti-TNF antibodies exerted a specific neutralizing effect. Lung permeability indices in rats that received sterile saline in place of nonspecific IgG or preimmune serum were all similar ( $<10 \%$ difference) suggesting that there was no suppression of lung injury attributable to a nonspecific "protective effect" of protein instilled into the lungs (data not shown). To confirm that intratracheal instillation of anti-TNF neutralized intrapulmonary TNF, we measured BAL fluid TNF activity $4 \mathrm{~h}$ after lung injury was induced in the presence of the blocking antibody preparation. Instillation of intratracheal anti-TNF resulted in nearly complete neutralization of intrapulmonary (BAL) TNF activity (Fig. 6).

Effect of added TNF on the development of lung injury. Recombinant mouse TNF $\alpha$ was instilled into the lungs of rats in order to determine whether exogenous TNF directly induces lung injury. Instillation of mouse TNF quantities equivalent to the maximum endogenous TNF activities measured in BAL fluid during evolving immune complex alveolitis resulted in modest increases in pulmonary vascular permeability (Table II). Histologic examination of lung sections revealed small, scattered foci of intraalveolar hemorrhage and neutrophil accumulation within alveolar septae (data not shown). Mouse TNF was also mixed with anti-BSA to determine whether the addition of exogenous TNF exhances the development of immune complex alveolitis. In these experiments,

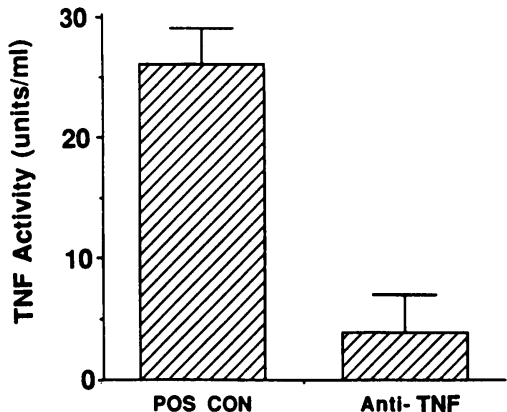

Figure 6. In vivo neutralization of intrapulmonary TNF activity. Anti-TNF $(30 \mu \mathrm{g})$ was instilled into rat lungs upon initiation of immune complex lung injury. Positive control rats received an equivalent concentration of either nonspecific rabbit IgG (shown in this figure) or preimmune rabbit serum $(200 \mu \mathrm{l})$ in place of anti-TNF. Negative control rats received intratracheal IgG anti-BSA but no intravenous BSA (not shown). BAL fluid TNF activities were measured after $4 \mathrm{~h}$ as described previously. These data represent the means \pm SEM from four rats in each group.

submaximal lung injury was induced by reducing the intratracheal anti-BSA from 250 to $80 \mu \mathrm{g}$. As shown in Table II, the addition of exogenous TNF to anti-BSA resulted in a small, dose-dependent augmentation of immune complex lung injury. These experiments provide additional evidence that TNF participates in the pathogenesis of acute immune complex alveolitis in the rat.

Evidence that TNF contributes to lung injury through a neutrophil-mediated mechanism. Transmission electron micrographs of lung sections from rats with immune complex alveolitis revealed the expected extensive intraalveolar hemorrhage and large neutrophil influx. In contrast, rats that received anti-TNF (30 $\mu \mathrm{g})$ exhibited a marked reduction in intraalveolar hemorrhage and a striking reduction in neutrophils (Fig. 7). Morphometric analysis of lung sections confirmed the diminished neutrophil influx and hemorrhagic changes in lungs of rats treated with anti-TNF (Table III).

Another method for measuring neutrophil content in lungs was also employed. Whole lungs from rats in each of the treatment groups were homogenized and assayed for myeloperoxidase activity (Table IV). There was a marked reduction in the myeloperoxidase activity present in lungs from rats treated with anti-cytokine antibodies compared with positive control animals. These data suggest that TNF is necessary for the recruitment of neutrophils into lungs of animals developing immune complex alveolitis.

Anti-TNF does not impair rat complement activation or chemotactic activity in activated serum. Measuring migrational responses of rat neutrophils, relevant concentrations of anti-TNF did not impair the chemotactic activity contained in rat serum that had been activated with zymosan (Table V). Similarly, incubation of anti-TNF (43 $\mu \mathrm{g})$ with fresh rat serum $(25 \mu \mathrm{l})$ did not impair complement activation by zymosan particles $(0.1 \mathrm{mg} / \mathrm{ml})$ as measured by rat neutrophil chemotaxis (data not shown). These experiments provide evidence that the anti-TNF antibody preparation does not interfere with

Figure 7. Evidence that anti-TNF leads to reduced intrapulmonary neutrophil influx during immune complex alveolitis. In positive control rats (intratracheal nonspecific rabbit IgG; $30 \mu \mathrm{g}$ ) there is extensive lung injury manifested by hemorrhage, neutrophil influx, interstitial edema and intraalveolar fibrin deposition $(A$ and $C)$. Rats that received anti-TNF $(30 \mu \mathrm{g})$ exhibit markedly reduced lung injury $(B$ and $D) . A$ and $B$ are $\times 250$ toluidine blue stained $1 \mu \mathrm{m}$ plastic sections. $C$ and $D$ are uranyl acetate and lead citrate stained transmission electron micrographs; $C$ $\times 4025 ; D \times 4950$. 

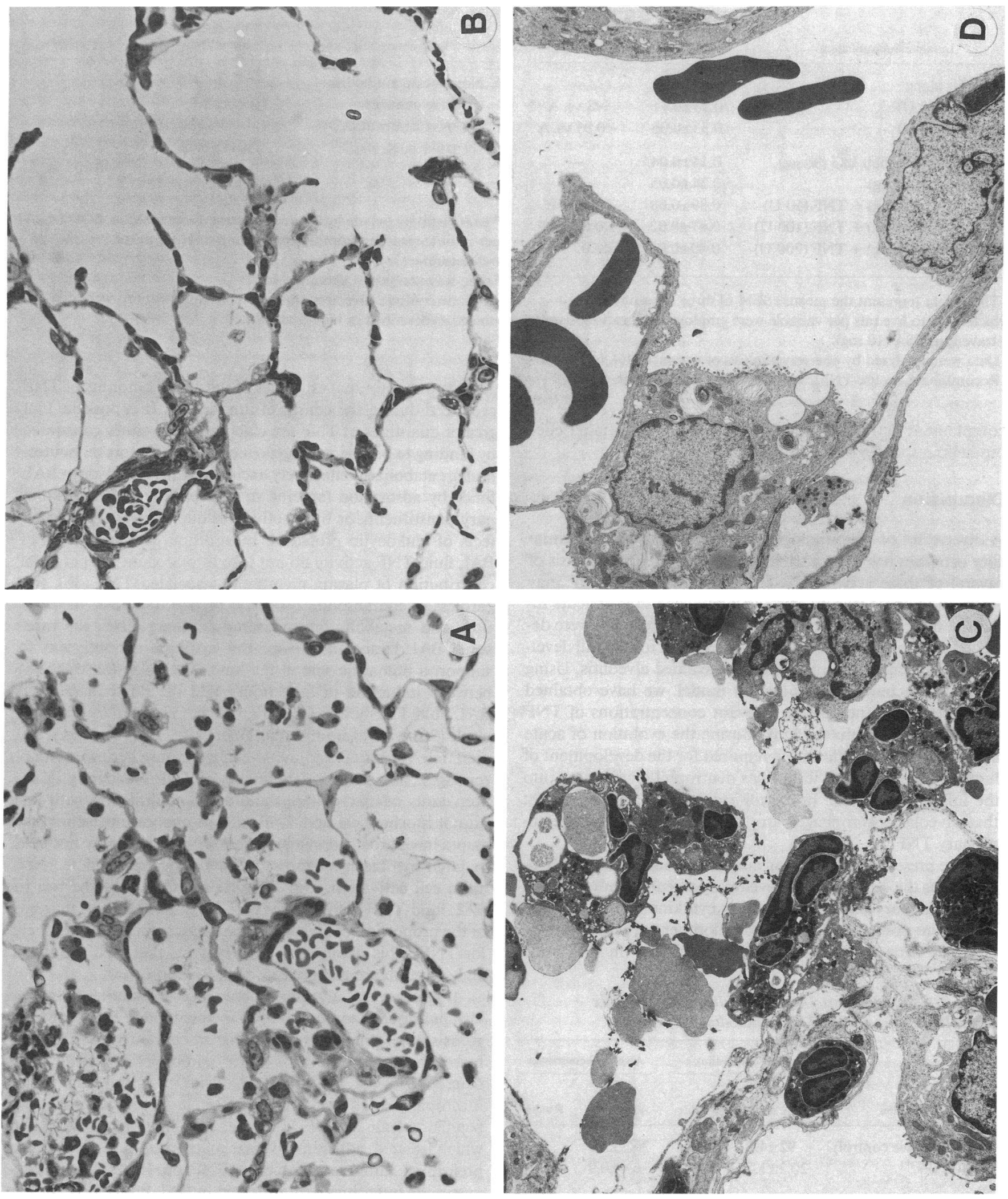
Table II. Effect of Exogenous TNF on the Development of Lung Injury

\begin{tabular}{lcc}
\hline & \multicolumn{2}{c}{ Lung injury } \\
\cline { 2 - 3 } & $\begin{array}{c}\text { Permeability index } \\
( \pm S E M)^{*}\end{array}$ & $P$ value \\
\hline \multicolumn{1}{c}{ Intratracheal instillation } & $0.09 \pm 0.04$ & - \\
A. Buffer alone & $0.23 \pm 0.11$ & NS vs. A \\
B. TNF $(100 \mathrm{U})^{\S}$ & $0.33 \pm 0.06$ & $<0.05$ vs. A \\
C. TNF $(200 \mathrm{U})$ & $0.14 \pm 0.04$ & - \\
D. Nonspecific rabbit IgG $(80 \mu \mathrm{g})$ & $0.34 \pm 0.05$ & - \\
E. Anti-BSA $(80 \mu \mathrm{g})$ & $0.39 \pm 0.08$ & $\mathrm{NS}$ vs. E \\
F. Anti-BSA $(80 \mu \mathrm{g})+\mathrm{TNF}(50 \mathrm{U})$ & $0.47 \pm 0.02$ & $<0.05$ vs. E \\
G. Anti-BSA $(80 \mu \mathrm{g})+\mathrm{TNF}(100 \mathrm{U})$ & $0.49 \pm 0.03$ & $<0.05$ vs. E \\
H. Anti-BSA $(80 \mu \mathrm{g})+\mathrm{TNF}(200 \mathrm{U})$ & 0.49
\end{tabular}

* These data represent the means \pm SEM of three experiments in which three to five rats per variable were employed. All rats received intravenous BSA (10 mg).

${ }^{\ddagger}$ Data were analyzed by one-way analysis of variance (46).

$\S$ Recombinant mouse TNF $\alpha$ was used in all experiments.

complement activation or with complement-mediated chemotactic responses of neutrophils.

\section{Discussion}

A diverse set of immunologic, metabolic, and proinflammatory activities has been attributed to TNF (1). On the basis of several of these activities, it can be inferred that TNF may function in anatomically compartmentalized sites of acute inflammation. The experiments described in this study were designed to determine whether TNF is required for the full development of acute immune complex-mediated alveolitis. Using a well characterized experimental model, we have obtained evidence that pathologically relevant concentrations of TNF are elaborated within the lungs during the evolution of acute alveolitis, that this cytokine is required for the development of lung injury, and that it influences neutrophil recruitment into the inflammatory site. Immunohistochemical studies suggest that alveolar macrophages are the chief source of intrapulmonary TNF.

The presence of increasing concentrations of TNF in BAL fluid over the 4-h course of developing alveolitis indicates that intrapulmonary elaboration of this cytokine occurs within a time frame compatible with the development of acute immune complex lung injury. Measurements of TNF in BAL fluid

Table III. Morphometric Analysis of Immune Complex Alveolitis: Effect of Anti-TNF Antibodies*

\begin{tabular}{|c|c|c|c|c|}
\hline \multirow[b]{2}{*}{ Intervention } & \multicolumn{2}{|c|}{ Neutrophil influx } & \multicolumn{2}{|c|}{ Alveolar hemorrhage } \\
\hline & $\begin{array}{c}\text { PMN/ } \\
\text { 40X HPF }\end{array}$ & $P$ value & $\begin{array}{c}\text { RBC/ } \\
\text { 40X HPF }\end{array}$ & $P$ value $^{\ddagger}$ \\
\hline None (positive control) & $92 \pm 4.6$ & - & $26.7 \pm 2.8$ & - \\
\hline Anti-TNF & $20.2 \pm 1.7$ & $<10^{-6}$ & $6.4 \pm 0.9$ & $<10^{-6}$ \\
\hline
\end{tabular}

\footnotetext{
* Immune complex alveolitis and anti-TNF interventions were conducted as described in Figs. 5 and 7.

‡ One-way analysis of variance (46).
}

Table IV. Neutrophil Influx into Lungs in IgG Immune Complex Alveolitis: Whole Lung Myeloperoxidase Activity*

\begin{tabular}{|c|c|c|}
\hline \multirow[b]{2}{*}{ Intervention } & \multicolumn{2}{|c|}{ Myeloperoxidase activity } \\
\hline & $\begin{array}{c}\text { Mean }( \pm \mathrm{SD}) \\
(n=3)\end{array}$ & $P$ value ${ }^{q}$ \\
\hline \multirow{2}{*}{$\begin{array}{l}\text { A Nonspecific antibodies } \\
\text { (positive control) } \\
\text { B No intravenous BSA } \\
\text { (negative control) }\end{array}$} & $0.411 \pm 0.09$ & - \\
\hline & $0.019 \pm 0.02$ & - \\
\hline C Anti-TNF & $0.173 \pm 0.05$ & $<0.005$ vs. A \\
\hline \multicolumn{3}{|c|}{$\begin{array}{l}\text { * MPO activity (whole lung homogenates) is expressed as } \triangle \mathrm{OD}\left(A_{460}\right) \\
\text { per minute resulting from decomposition of } \mathrm{H}_{2} \mathrm{O}_{2} \text { in the presence of } \\
0 \text {-dianisidine (38). } \\
\text { } \text { One-way analysis of variance (46). } \\
\text { Immune complex alveolitis and anti-TNF interventions were con- } \\
\text { ducted as described in Figs. } 5 \text { and } 7 \text {. }\end{array}$} \\
\hline
\end{tabular}

provide only minimal estimates of the actual amounts of TNF produced during the course of lung injury. It is possible that greater quantities of TNF are elaborated but either consumed by binding to biological targets (including receptors on neutrophils), catabolized, effectively excluded from retrieval (in BAL fluid) by adsorption (specific or nonspecific) to intrapulmonary constituents or by inactivation due, for example, to effects of leukocytic proteases. In addition, measurements of BAL fluid TNF activity do not take into account the potential contribution of plasma membrane-associated TNF (36). Regardless of the absolute quantity, precise anatomic distribution, and metabolic fate of intrapulmonary TNF, the measured BAL concentrations of this cytokine are sufficient to support a phlogistic role in the lung. The delay (at least $2 \mathrm{~h}$ ) between induction of lung injury and the rapid increase in BAL fluid TNF activity is similar to that reported by Chensue et al. (36) who examined the kinetics of TNF synthesis and secretion by Freund's adjuvant-elicited mouse peritoneal macrophages. Based on measurements of TNF bioactivity in supernatants of macrophage cultures, in conjunction with immunohistochemical and TNF mRNA analyses, these investigators have provided evidence that TNF is probably not held in large cytoplasmic stores but is synthesized and secreted upon cell activation. Our kinetic data show that the rise in BAL fluid TNF activity over time is paralleled by an increase in the proportion of alveolar macrophages that stain positively for TNF. This observation also suggests that TNF synthesis and secretion are triggered by cell activation.

It appears that alveolar macrophages constitute the major intrapulmonary source of TNF in this model. Based on previous studies, the lungs of a 350-g rat contain $\sim 10^{7}$ alveolar macrophages and 1.5 to $4 \times 10^{6}$ additional interstitial lung macrophages $(42,43)$. In vitro activation of isolated alveolar macrophages with IgG immune complexes results in dose-dependent release of TNF. Under idealized in vitro conditions in which isolated, adherent alveolar macrophages are exposed to preformed immune complexes, TNF production easily surpasses the quantities demonstrated in BAL fluids. It is unclear to what extent the total intrapulmonary TNF pool is determined by the proportion of activated (TNF secreting) macrophages versus the quantity of TNF secreted per cell. Studies 
Table V. Effect of Anti-TNF Antibodies on Neutrophil Chemotaxis

\begin{tabular}{lcc}
\hline & \multicolumn{2}{c}{ Locomotion index ${ }^{*}(\mu \mathrm{m})$} \\
\cline { 2 - 3 } Condition & Experiment 1 & Experiment 2 \\
\hline Unactivated rat serum $(25 \mu \mathrm{l})$ & $14 \pm 6$ & $24 \pm 6$ \\
$\begin{array}{l}\text { Activated rat serum }(25 \mu \mathrm{l}) \\
\text { plus HBSS alone }\end{array}$ & $57 \pm 8$ & $82 \pm 11$ \\
plus irrelevant rabbit IgG $(330 \mu \mathrm{g})$ & $51 \pm 6$ & $78 \pm 6$ \\
plus anti-TNF $(43 \mu \mathrm{g})$ & $51 \pm 5$ & $81 \pm 5$ \\
plus anti-TNF $(29 \mu \mathrm{g})$ & $56 \pm 8$ & $74 \pm 9$ \\
plus anti-TNF $(14 \mu \mathrm{g})$ & $52 \pm 9$ & $77 \pm 12$ \\
& & \\
\hline
\end{tabular}

* Locomotion index (mean \pm SD) was calculated using a modified micropore filter assay for granulocyte leukotaxis $(39,40)$. Duplicate wells contained the chemotactic stimulus (serum) in a final volume of $225 \mu \mathrm{l}$ HBSS. The neutrophil chambers contained $1.5 \times 10^{6} / \mathrm{ml}$ PMN in $333 \mu \mathrm{l}$ of HBSS with $1 \%$ BSA.

Serum was activated with $0.1 \mathrm{mg} / \mathrm{ml}$ zymosan for $30 \mathrm{~min}$ at $37^{\circ} \mathrm{C}$.

focusing on cell activation and the regulation of TNF synthesis and secretion will be required to sort out these issues.

Direct analysis of the pathogenetic role of TNF in immune complex alveolitis is predicted on the ability to selectively neutralize rat TNF activity. To this end, neutralizing antibodies were raised in rabbits immunized with recombinant mouse TNF $\alpha$. The specificity of anti-TNF has been confirmed by Western immunoblot analysis. Subsequent in vitro and in vivo neutralization studies have provided evidence that this reagent specifically neutralizes rat TNF, that it does not cross react with IL-1, and that it neutralizes TNF within rat lungs as determined by analysis of BAL fluid.

The in vivo studies summarized in Fig. 5 demonstrate that intrapulmonary instillation of anti-TNF results in marked attenuation of immune complex-mediated lung injury. The specificity and neutralizing capacity of anti-TNF, in conjunction with the lack of suppressive effects exhibited by control antibodies, strongly suggest that intrapulmonary TNF plays a major role in the pathogenesis of immune complex alveolitis. This conclusion is further supported by the observation that the intrapulmonary instillation of exogenous TNF, either alone or with anti-BSA, can recapitulate or amplify the lung injury associated with alveolar immune complex deposition.

Morphometric analysis of intraalveolar hemorrhage corroborates the pulmonary vascular permeability data and provides evidence that the differences in permeability between protected and unprotected rats reflect differences in lung injury, not solely net differences in transcapillary solute transport. Morphometric analysis revealed a striking reduction in neutrophils in lungs of rats treated with anti-TNF. Whole lung myeloperoxidase activity measurements confirmed the marked reduction in pulmonary neutrophil influx. The reduction in intraalveolar hemorrhage observed in rats treated with anti-TNF does not preclude the possibility that TNF directly influences transvascular protein clearance distinct from capillary disruption due to neutrophil-mediated tissue injury. Recent studies by Horvath et al. (23) indicate that infusions of TNF $\alpha$ into sheep result in increased pulmonary transvascular protein clearance through a mechanism independent of neutrophils. These investigators suggest that TNF may play a role in pulmonary microvascular leakage through neutrophil-inde- pendent process(es). Anti-TNF does not impair complement activation or the chemotactic activity present in zymosan-activated serum. These experiments provide evidence against the possibility that anti-TNF antibodies directly interfere with complement-mediated neutrophil recruitment.

The salient mechanism(s) involved in TNF mediated neutrophil influx are not known in this model, but several activities ascribed to this cytokine could play a role. While it is clear that TNF is linked to neutrophil influx, it is unknown whether this effect is direct or indirect. For instance, TNF is thought to be chemotactic for neutrophils (9) although this action is debated (44). TNF induces the expression of endothelial and neutrophil adhesion molecules $(11,22)$ which appear to be critical in directing neutrophils into inflammatory sites. TNF can trigger endothelial cells to produce platelet-activating factor (24), which in turn is chemotactic for neutrophils (45). Recently, Strieter et al. (25) have shown that TNF can induce the synthesis of a neutrophil chemotactic factor by several types of cells that are indigenous to the lung. It is possible that the requirement for TNF is related to the sequential actions of more than one cytokine. For instance, TNF can trigger IL-1 and IL- 6 production by endothelial cells and fibroblasts (1). In this scenario, neutralization of TNF would prevent elaboration of other cytokines. The present studies support the concept that locally elaborated TNF plays a major role in regulating a compartmentalized acute inflammatory response. Further investigation will be required to determine the precise relationships between TNF and other important proinflammatory mediator systems.

\section{Acknowledgments}

We thank The Upjohn Company for providing recombinant human IL-1 $\beta$. We are grateful to Kimberly D. Drake and Sue Maier for skillful preparation of the manuscript.

This work was supported in part by National Institutes of Health grants HL-40526, HL-31963, and GM-29507.

\section{References}

1. Sherry, B., and A. Cerami. 1988. Cachectin/tumor necrosis factor exerts endocrine, paracrine, and autocrine control of inflammatory responses. J. Cell Biol. 107:1269-1277.

2. Beutler, B., I. W. Milsark, and A. Cerami. 1985. Cachectin/ tumor necrosis factor: Production, distribution, and metabolic fate in vivo. J. Immunol. 135:3972-3977.

3. Tracey, K. J., Y. Fong, D. G. Hesse, K. R. Manogue, A. T. Lee, G. C. Kuo, S. F. Lowry, and A. Cerami. 1987. Anti-cachectin/TNF monoclonal antibodies prevent septic shock during lethal bacteraemia. Nature (Lond.). 330:662-664.

4. Beutler, B., I. W. Milsark, and A. Cerami. 1985. Passive immunization against cachectin/tumor necrosis factor protects mice from lethal effect of endotoxin. Science (Wash. DC). 229:869-871.

5. Tracey, K. J., B. Beutler, S. F. Lowry, J. Merryweather, S. Wolpe, I. W. Milsark, R. J. Harir, T. J. Fahey III, A. Zentella, J. D. Albert, G. T. Shires, and A. Cerami. 1986. Shock and tissue injury induced by recombinant human cachectin. Science (Wash. DC). 234:470-474.

6. Tracey, K. J., S. F. Lowry, T. J. Fahey III, J. D. Albert, Y. Fong, D. Hesse, B. Beutler, K. R. Manogue, S. Calvano, H. Wei, A. Cerami, and G. T. Shires. 1987. Cachectin/tumor necrosis factor induces lethal shock and stress hormone responses in the dog. Surg. Gynecol. Obstet. 164:415-422.

7. Remick, D. G., R. G. Kunkel, J. W. Larrick, and S. L. Kunkel. 1987. Acute in vivo effects of human recombinant tumor necrosis factor. Lab. Invest. 56:583-590. 
8. Mathison, J. C., E. Wolfson, and R. J. Ulevitch. 1988. Participation of tumor necrosis factor in the mediation of gram negative bacterial lipopolysaccharide induced injury in rabbits. J. Clin. Invest. 81:1925-1937.

9. Ming, W. J., L. Bersan, and A. Mantovani. 1987. Tumor necrosis factor is chemotactic for monocytes and polymorphonuclear leukocytes. J. Immunol. 138:1469-1474.

10. Klebanoff, S. J., M. A. Vadas, J. M. Harlan, L. J. Sparks, J. R. Gamble, J. M. Agosti, and A. M. Waltersdorph. 1986. Stimulation of neutrophils by tumor necrosis factor. J. Immunol. 136:4220-4225.

11. Gamble, J. R., J. M. Harlan, S. J. Klebanoff, and M. A. Vadas. 1985. Stimulation of the adherence of neutrophils to umbilical vein endothelium by human recombinant tumor necrosis factor. Proc. Natl. Acad. Sci. USA. 82:8667-8674.

12. Tsujimoto, M., S. Yokota, J. Vilek, and G. Weissman. 1986. Tumor necrosis factor provokes superoxide anion generation from neutrophils. Biochem. Biophys. Res. Commun. 137:1094-1100.

13. Bachwich, P. R., S. W. Chensue, J. W. Larrick, and S. W. Kunkel. 1986. Tumor necrosis factor stimulates interleukin-1 and prostaglandin $\mathrm{E}_{2}$ production in resting macrophages. Biochem. Biophys. Res. Commun. 136:94-101.

14. Philip, R., and L. B. Epstein. 1986. Tumor necrosis factor as immunomodulator and mediator of monocyte cytoxicity induced by itself, g-interferon and interleukin-1. Nature (Lond.). 323:86-89.

15. De Titto, E. H., J. R. Catterall, and J. S. Remington. 1986. Activity of recombinant tumor necrosis factor on Toxoplasma gondii and Trypanosoma cruzi. J. Immunol. 137:1342-1345.

16. Warren, J. S., S. L. Kunkel, T. W. Cunningham, K. J. Johnson, and P. A. Ward. 1988. Macrophage-derived cytokines amplify immune complex-triggered $\mathrm{O}_{2}^{-}$responses by rat alveolar macrophages. Am. J. Pathol. 130:489-495.

17. Nawroth, P. P., and D. M. Stern. 1986. Modulation of endothelial cell hemostatic properties by tumor necrosis factor. J. Exp. Med. 163:740-745.

18. Bevilacqua, M. P., J. S. Pober, G. R. Majeau, W. Fiers, R. S. Cotran, and M. A. Gimbrone, Jr. 1986. Recombinant tumor necrosis factor induces procoagulant activity in cultured human vascular endothelium: characterization and comparison with the actions of interleukin 1. Proc. Natl. Acad. Sci. USA. 83:4533-4537.

19. Nawroth, P. P., I. Bank, D. Handley, J. Cassimeris, L. Chess, and D. Stern. 1986. Tumor necrosis factor/cachectin interacts with endothelial cell receptors to induce release of interleukin 1. J. Exp. Med. 163:1363-1375.

20. Libby, P., J. M. Ordovas, K. R. Auger, A. H. Robbins, L. K. Biriyi, and C. A. Dinarello. 1986. Endotoxin and tumor necrosis factor induce interleukin-1 gene expression in adult human vascular endothelial cells. J. Pathol. 124:179-185.

21. Bevilacqua, M. P., J. S. Pober, G. R. Majeau, R. S. Cotran, and M. A. Gimbrone, Jr. 1984. Interleukin 1 (IL-1) induces biosynthesis and cell surface expression of procoagulant activity in human vascular endothelial cells. J. Exp. Med. 160:618-623.

22. Pohlman, T. H., K. A. Stanness, P. G. Beatty, H. D. Ochs, and J. M. Harlan. 1986. An endothelial cell surface factor(s) induced in vitro by lipopolysaccharide, interleukin 1 , and tumor necrosis factor- $\alpha$ increases neutrophil adherence by a CDw18-dependent mechanism. $J$. Immunol. 136:4548-4553.

23. Horvath, C. J., T. J. Ferro, G. Jesmok, and A. B. Malik. 1988. Recombinant tumor necrosis factor increases pulmonary vascular permeability independent of neutrophils. Proc. Natl. Acad. Sci. USA. 85:9219-9223.

24. Bussolino, F., G. Camussi, and C. Baglioni: Synthesis and release of platelet activating factor by human vascular endothelial cells treated with TNF or IL-1 $\alpha$. J. Biol. Chem. 263:11856-11861.

25. Strieter, R. M., S. L. Kunkel, H. Showell, D. G. Remick, S. H. Phan, P. A. Ward, and R. M. Marks. 1989. Human endothelial cell gene expression of a neutrophil chemotactic factor by TNF-a, LPS, and IL-1b. Science (Wash. DC). 243:1467-1469.

26. Movat, H. Z., C. E. Burrowes, M. I. Cybulsky, and C. A.
Dinarello. 1987. Acute inflammation and a Schwartzman-like reaction induced by interleukin 1 and tumor necrosis factor: synergistic action of the cytokines in the induction of inflammation and microvascular injury. Am. J. Pathol. 129:463-471.

27. Issekutz, A. C., and Z. Wankowicz. 1987. Synergy between tumor necrosis factor (TNF- $\alpha$ ) and interleukin 1 (IL-1) in the induction of polymorphonuclear leukocyte (PMNL) emigration. Fed. Proc. 46:737a.

28. Cybulsky, M. I., D. J. McComb, and H. Z. Movat. 1988. Neutrophil leukocyte emigration induced by endotoxin mediator roles of interleukin-1 and tumor necrosis factor. J. Immunol. 140:3144-3149.

29. Sharpe, R. J., R. J. Margolis, E. P. Amento, and R. D. Granstein. 1987. Induction of dermal acute inflammation by tumor necrosis factor. J. Invest. Dermatol. 88:517A. (Abstr.)

30. Johnson, K. J., and P. A. Ward. 1974. Acute immunologic pulmonary alveolitis. J. Clin. Invest. 54:349-357.

31. Johnson, K. J., and P. A. Ward. 1981. Role of oxygen metabolites in immune complex injury of lung. J. Immunol. 126:2365-2369.

32. Ward, P. A., R. E. Duque, M. C. Sulavik, and K. J. Johnson. 1983. In vitro and in vivo stimulation of rat neutrophils and alveolar macrophages by immune complexes. Production of $\mathrm{O}_{2}^{-}$and $\mathrm{H}_{2} \mathrm{O}_{2}$. Am. J. Pathol. 110:297-309.

33. Ward, P. A., G. O. Till, R. Kunkel, and C. Beauchamp. 1983. Evidence for role of hydroxyl radical in complement and neutrophil dependent tissue injury. J. Clin. Invest. 72:789-797.

34. Ruff, M. R., and G. E. Gifford. 1980. Purification and physicochemical characterization of rabbit tumor necrosis factor. J. Immunol. 125:1671-1677.

35. Mizel, S. 1981. Production and quantitation of lymphocyte activating factor (interleukin 1). In Manual of Macrophage Methodology. H. B. Herscowitz, H. T. Holden, J. A. Bellanti, and A. Ghaffar, editors. Marcel Dekker, New York 329-336.

36. Chensue, S. W., D. G. Remick, C. Shmyr-Forsch, T. F. Beals, and S. L. Kunkel. 1988. Immunohistochemical demonstration of cytoplasmic and membrane-associated tumor necrosis factor in murine macrophages. Am. J. Pathol. 133:564-572.

37. Laemmli, U. K. 1970. Cleavage of structural proteins during the assembly of the bacteriophage T4. Nature (Lond.). 227:680-685.

38. Henson, P. M., B. Zanolari, N. A. Schwartzman, and S. R Hong. 1978. Intracellular control of human neutrophil secretion: I. C5a-induced stimulus-specific desensitization and the effects of cytochalasin B. J. Immunol. 121:851-855.

39. Boyum, A. 1968. Separation of leukocytes from blood and bone marrow. Scand. J. Clin. Lab. Invest. 21:77-84.

40. Zigmond, S. H., and J. G. Hirsch. 1973. Leucocyte locomotion and chemotaxis. New methods for evaluation and demonstration of a cell-derived chemotactic factor. J. Exp. Med. 137:387-394.

41. Maderazo, E. G., and C. L. Woronick. 1987. A modified micropore filter assay of human granulocyte chemotaxis. In Leukocyte Chemotaxis. J. I. Gallin, and P. G. Quie, editors. Raven Press, New York.

42. Lehnert, B. E., Y. E. Valdez, and L. M. Holland. 1985. Pulmonary macrophages: alveolar and interstitial populations. Exp. Lung Res. 9:177-186.

43. Warren, J. S., R. G. Kunkel, K. J. Johnson, and P. A. Ward. 1987. Comparative $\mathrm{O}_{2}^{-}$responses of lung macrophages and blood phagocytic cells in the rat. Possible relevance to IgA immune complex induced lung injury. Lab. Invest. 57:311-320.

44. Mrowietz, U., J. M. Schroder, and E. Christophers. 1988. Recombinant human tumor necrosis factor lacks chemotactic activity for human peripheral blood neutrophils and monocytes. Biochem. Biophys. Res. Commun. 153:1223-1228.

45. Humphrey, D. M., D. J. Hanahan, and R. N. Pinckard. 1982. Induction of leukocytic infiltration in rabbit skin by acetyl glyceryl either phosphorylcholine. Lab. Invest. 47:227-234.

46. Linton, M., and P. S. Gallo, Jr. 1975. The practical statistician: simplified handbook of statistics. Brooks-Cole Publishing Co., Monterey, CA. 136-218. 